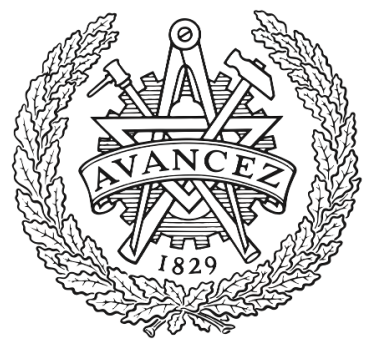

CHALMERS

UNIVERSITY OF TECHNOLOGY

\title{
Single Dark-Pulse Kerr Comb Supporting 1.84 Pbit/s Transmission over 37-Core Fiber
}

Downloaded from: https://research.chalmers.se, 2023-04-26 14:29 UTC

Citation for the original published paper (version of record):

Kong, D., Jorgensen, A., Henriksen, M. et al (2020). Single Dark-Pulse Kerr Comb Supporting 1.84 Pbit/s Transmission over 37-Core Fiber. Conference Proceedings - Lasers and Electro-Optics Society Annual Meeting-LEOS, 2020-May. http://dx.doi.org/10.1364/CLEO_AT.2020.JTh4A.7

N.B. When citing this work, cite the original published paper. 


\title{
Single Dark-Pulse Kerr Comb Supporting 1.84 Pbit/s Transmission over 37-Core Fiber
}

\author{
D. Kong' ${ }^{1}$ A. A. Jørgensen ${ }^{2}$, M. R. Henriksen ${ }^{2}$, F. Klejs ${ }^{1}$ Z. Ye ${ }^{3}$, Ò. B. Helgason ${ }^{3}$, H. E. Hansen ${ }^{1}$, H. Hu ${ }^{1}$, M. \\ Yankov $^{1}$, S. Forchhammer' , P. Andrekson ${ }^{3}$, A. Larsson ${ }^{3}$, M. Karlsson ${ }^{3}$, J. Schröder ${ }^{3}$, Y. Sasaki ${ }^{4}$, K. Aikawa ${ }^{4}$, \\ J.W. Thomsen ${ }^{2}$, T. Morioka ${ }^{1}$, M. Galili' ${ }^{1}$, V. Torres-Company ${ }^{3}$ and L. K. Oxenløwe ${ }^{1}$ \\ ${ }^{\prime}$ DTU Fotonik, Technical University of Denmark, Ørsteds Plads 343, DK-2800 Kgs. Lyngby, Denmark \\ ${ }^{2}$ Niels Bohr Institute, University of Copenhagen, Blegdamsvej 17, 2100 Copenhagen Ø, Denmark \\ ${ }^{3}$ Photonics Laboratory, Department of Microtechnology and Nanoscience, Chalmers University of Technology, Gothenburg, Sweden. \\ ${ }^{4}$ Optical Technologies R\&D Center, Fujikura Ltd., 1440, Mutsuzaki, Sakura, Chiba, 285-8550, Japan \\ asbjorn.arvad@nbi.ku.dk
}

\begin{abstract}
We show that a single dark-pulse Kerr comb can generate high enough OSNR to carry $1.84 \mathrm{Pbit} / \mathrm{s}$ data, achieved by $223 \mathrm{WDM}$ spectral lines modulated with 32-Gbaud, SNR-adapted probabilistically shaped DP-QAM, over a 37-core fiber. (C) 2020 The Authors
\end{abstract}

Optical frequency combs show potential as sources for future communication systems. The intrinsic relative line stability of the combs can enable joint digital signal processing, which could lead to below zero guard-bands [1], easier phase tracking [2], and improved nonlinear transmission [3]. Furthermore, the large bandwidths of combs have allowed fiber-based comb sources to support data rates of several Pbit/s [4,5]. Nonlinear microring resonator (MRR) based optical frequency combs provide the same advantageous characteristics of traditional combs while maintaining a small footprint and integrateability. While two MRRs producing bright soliton combs were used to carry 50 Tbit/s [6], another class of MRRs producing dark-pulse Kerr combs [7] have become attractive due to its 5-10 times larger pump-to-comb conversion efficiency [8], allowing for higher-order data modulation formats [9].

We report on an MRR dark-pulse Kerr comb covering the C+L-bands, with an average OSNR per line of $27 \mathrm{~dB}$ in $0.1 \mathrm{~nm}$ bandwidth. We use this comb as the source of light to demonstrate $1.84 \mathrm{Pbit} / \mathrm{s}$ achieved data rate transmission through a 7.9-km 37-core single-mode fiber, the highest data rate carried by light from a microring comb source.
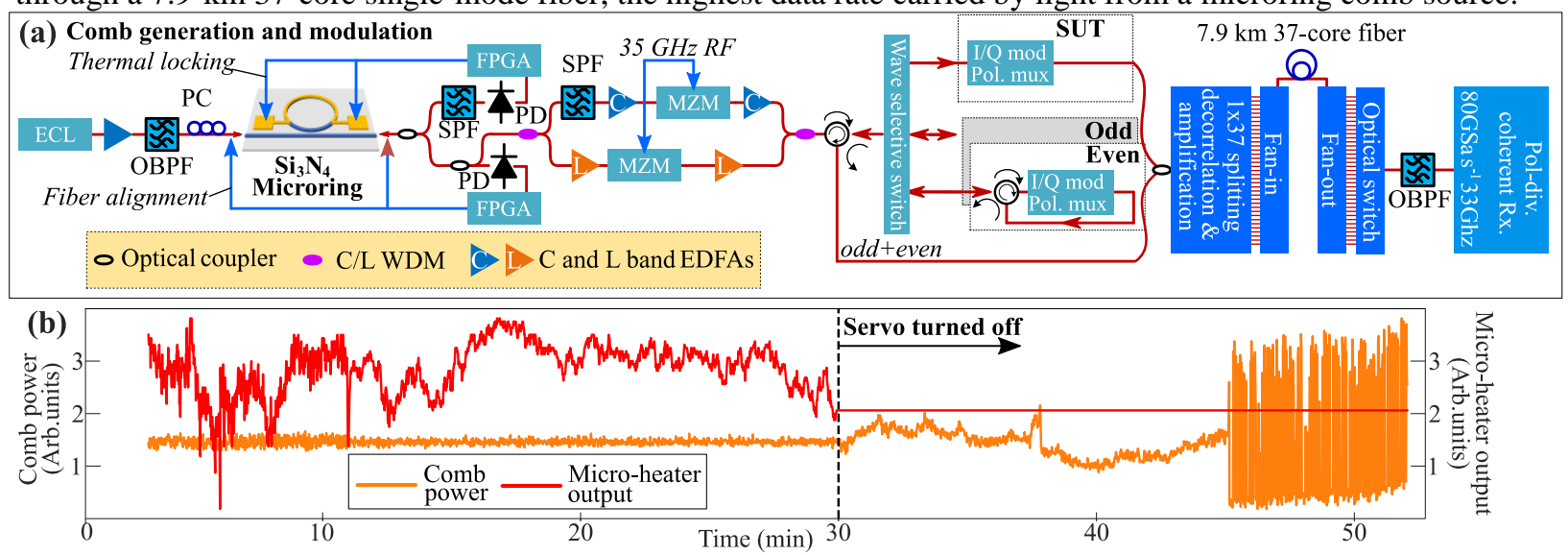

Fig. 1. (a) The experimental setup. (b) Measured comb power (Orange) and on-chip micro heater output (Red) during a timeperiod where the control servo is switched off after $30 \mathrm{~min}$.

We use a $105.2 \mathrm{GHz}$ free spectral range (FSR) $\mathrm{Si}_{3} \mathrm{~N}_{4}$ MRR to generate a dark-pulse Kerr comb. The device is fabricated using a novel subtractive processing method [10] and has a mean intrinsic Q of 10.6 million. The width and height of the ring waveguide are $1850 \mathrm{~nm}$ and $600 \mathrm{~nm}$, respectively. Its over-coupled design optimizes the conversion efficiency [8] (17\% measured for $29 \mathrm{dBm}$ of on-chip coupled power). The measured dispersion is $68 \mathrm{ps}^{2} / \mathrm{km}^{2}$, designed to maximize the power per line across the available bandwidth [8]. The pump laser is set at $1562.7 \mathrm{~nm}$, close to resonance with a weak mode crossing [10]. The dark pulse Kerr comb is initialized using an on-chip microheater to tune the resonance closer to the pump, i.e. with fixed pump wavelength.

The experimental setup is shown in Fig. 1(a). Leaving the MRR, the comb frequencies are divided into the $\mathrm{C}$ and L bands. The pump is filtered away by a short pass filter (SPF), at the cost of 3 spectral lines. An FPGA-based servo uses the microheater to stabilize the optical power in the generated comb lines. This is critical for long term operation as seen in Fig. 1(b) where the optical comb power is plotted together with the micro-heater output. This setup can maintain the comb state for several hours. The line spacing stability is measured by electrooptic downconversion and 
displays a modest frequency drift of less than $1 \mathrm{MHz}$ over days.

To match the linespacing of the comb with our transmitter and receiver subsystems, the original comb lines are multiplied using Mach-Zehnder amplitude modulators (MZMs), one in each band, yielding $35 \mathrm{GHz}$ spaced lines. The 35-GHz modulated comb is data modulated at 32 Gbaud in a standard odd, even, signal-under-test (SUT) scheme [11]. The signals are polarization-division multiplexed (PDM) using a PDM emulator with 1887-symbol delay and digitally pulse shaped by a root-raised cosine filter with 401 taps and roll-off factor of 0.01 . The modulation is probabilistically shaped 64- or 256-QAM constellations with a Maxwell-Boltzmann distribution and generated using a distribution matcher [12]. The shaping is tailored to the measured SNR of the SUT channel [13], adapted to the worst core. All 37 cores use this shaping and are expected to achieve the same data rate. LDPC coding with 50\% and 33\% overheads are used for the 64- and 256-QAM signals. These overheads were determined by the constellation shaping and distribution matcher implementation. All L-band channels and six C-band channels use 64 QAM while the rest uses 256 QAM. The C- and L-band EDFAs and the waveshaper used in this experiment give an upper limit of 9-THz available bandwidth. In total, 223 channels (121 in the $\mathrm{C}$ band and 102 in the L band) are generated and successfully transmitted. The WDM channels are decorrelated and launched into the 7.9-km 37-core fiber [14-15]. The SUT channel is extracted by an optical switch and an optical bandpass filter (OBPF) and detected with a coherent receiver.

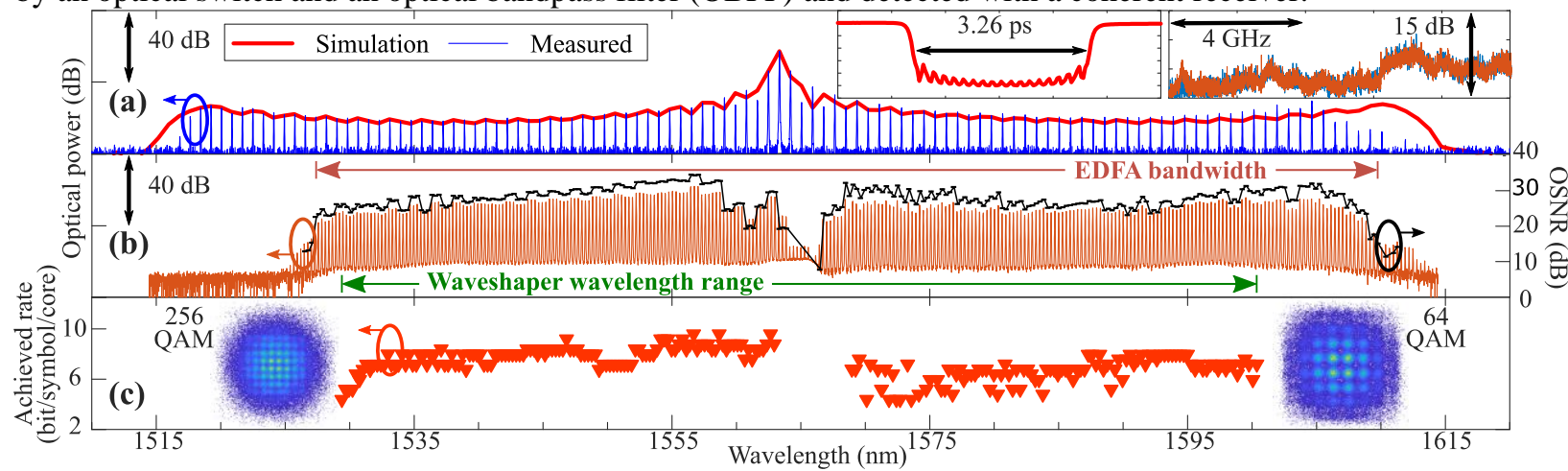

Fig. 2. (a) Optical spectra of the simulation (red) and the measured dark-pulse Kerr comb (blue). Insets show the simulated time-domain pulse profile and the measured RF beat spectrum of the comb from DC to $10 \mathrm{GHz}$; (b) Optical spectrum (brown) of the 35-GHz modulated comb with measured OSNR of each line (black); (c) Achieved bit rate in bit per symbol per fiber-core, after FEC decoding with error-free performance. All 37 cores achieve the same data rate for each channel by design. Insets are representative probabilistically shaped constellation maps for the received 256- and 64-QAM signals.

Figure 2(a) shows the spectrum of the comb. The insets show the simulated time-domain profile and the measured RF amplitude noise spectrum, both confirming mode-locking of the comb. The final output spectrum of our source is depicted in Fig. 2(b) together with the OSNR measured before the first circulator. Figure 2(c) shows the experimentally achieved bit rate in terms of bit per symbol per core. Each point represents 37 cores as they were all tested with identical bit rates based on the tailored shaping. The two insets are representative probabilistically shaped constellations for the received 256- and 64-QAM signals. The performance degradation in the lower $\mathrm{C}$ band is due to the EDFA gain shape limitation. The performance variation in the $\mathrm{L}$ band comes from the instabilities of the L-band EDFAs used in this experiment. In all, 121 channels in the C band (6 channels of 64 QAM and 115 of 256 QAM) and 102 channels in the L band (64 QAM) are successfully transmitted. The total achieved bit rate excluding the LDPC overhead is $1.93 \mathrm{Pbit} / \mathrm{s}$. A data rate of $1.84 \mathrm{Pbit} / \mathrm{s}$ is calculated after subtracting the $4 \%$ pilot overhead and the assumed $1 \%$ outer FEC overhead used to correct any remaining errors (BER below $10^{-5}$ ) from the LDPC decoder [16].

We have demonstrated the highest reported data rate carried on the light from a single microring comb source, reaching 1.84 Pbit/s on 223 WDM channels over a 37-core fiber.

This work is partly supported by the DNRF Research CE SPOC (ref. DNRF123), the Swedish Research Council (grant no. 2016-06077, project iTRAN), the ERC CoG Grant 771410, the EU-Japan coordinated R\&D project SAFARI supported by the MIC of Japan and EC Horizon 2020. The H2020 Marie Skoldowska Curie ITN (GA 812818), the Swedish Research Council (VR) grants VR-2015-00535, 2016-06077, 2016-03960

\section{References}

[1] M. Mazur et al., arXiv:1911.02326v2 (2019)

[2] L. Lundberg et al., Nat. Comm. 11, 201 (2020).

[3] E. Temprana et al., J. Lightwave Technol. 34, 3544-3549 (2016).

[4] B. J. Puttnam et al,. in ECOC, p. PDP.3.1 (2015).

[5] G. Rademacher et al., in OFC, p. Th3H.1 (2020).

[6] P. Marin-Palomo et al., Nature. 546, 274-279 (2017).

[7] X. Xue et al., Nat. Photonics 9, 594-600 (2015).

[8] Ó. B. Helgason et al., J. Opt. Soc. Am. B 36(8), 2013 (2019).
[9] A. Fülöp et al., Nat. Comm. 9, 1598 (2018).

[10] Z. Ye et al., Opt. Express 27, 35719-35727 (2019).

[11] D. Kong et al., in ECOC, p. Th3F.5 (2018).

[12] G. Böcherer et al., IEEE Trans. Commun., 63(12), 2015.

[13] E. P. A. Silva et al, in ECOC p. Th1D (2018)

[14] Y. Sasaki et al., in OFC, p. Th1H.2 (2017).

[15] T. Kobayashi et al, in $O F C$, p. Th5B.1 (2017).

[16] D. S. Millar et al., J. Lightwave Technol.34, 1453-1463 (2016) 